\title{
ORIENTACIÓN PROFESIONAL EN LA EDUCACIÓN MEDIA Y DESARROLLO
} LOCAL

AUTORES: Claudia Miranda Pérez ${ }^{1}$

Ariel Caridad Reyes Torres ${ }^{2}$

Isbel Barrera Cabrera ${ }^{3}$

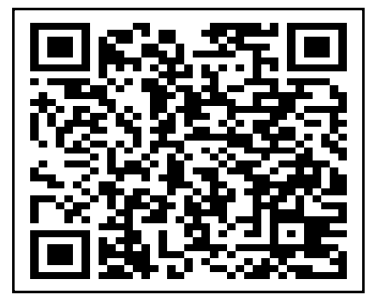

DIRECCIÓN PARA CORRESPONDENCIA: (claudia.miranda9311@nauta.cu )

Fecha de recepción: 12/06/2021

Fecha de aceptación: 22/12/2021

\section{RESUMEN}

La necesidad de una orientación profesional que permita lograr la matrícula de los estudiantes en las diferentes especialidades y carreras, en correspondencia con el desarrollo de la economía y la sociedad, fundamentalmente, incrementar la matrícula en carreras agropecuarias, pedagógicas, tecnológicas y de ciencias básicas afines. Por tales razones reforzar, de conjunto con las entidades de la producción y los servicios, las organizaciones políticas, estudiantiles y de masas y con la participación de la familia, la formación vocacional y orientación profesional, desde la primaria. Por ello los profesores de la enseñanza media tiene como reto desarrollar un proceso de orientación profesional en la escuela, que responda a las demandas del territorio, para formar a los profesionales que son fundamentales para su desarrollo. Por tanto, el proceso educativo en los diferentes centros educacionales, debe contribuir a la formación de la vocación, intereses y motivaciones en los estudiantes, por aquellas profesiones que respaldan los sectores estratégicos.

PALABRAS CLAVE: Orientación profesional; demandas del territorio; desarrollo; formación de la vocación; intereses y motivaciones.

\section{PROFESSIONAL ORIENTATION IN SECONDARY EDUCATION AND LOCAL DEVELOPMENT}

\section{ABSTRACT}

The need for a professional orientation that allows students to enroll in different specialties and careers, in correspondence with the development of the economy and society, fundamentally, increase the enrollment of agricultural, pedagogical, technological and basic science careers related. For these reasons, to reinforce, together with the entities of production and services, political, student and mass organizations and with the participation of the family, vocational training and professional orientation, from primary school. For this reason, secondary school teachers have the challenge of developing a process of professional guidance in the school, which

\footnotetext{
${ }^{1}$ Dirección Provincial de Educación, Pinar del Río. Cuba. E-mail: claudia.miranda9311@nauta.cu .

${ }^{2}$ Universidad de Pinar del Río. Hermanos Saiz Monte de Oca. Pinar del Río. Cuba.

${ }^{3}$ Universidad de Pinar del Río. Hermanos Saiz Monte de Oca. Pinar del Río. Cuba.
} 
responds to the demands of the territory, to train the professionals who are essential for their development. Therefore, the educational process in the different educational centers must contribute to the formation of vocation, interests and motivations in students, for those professions that support the strategic sectors

KEYWORDS: Professional orientation, demands of the territory, development, vocational training, interests and motivations

\section{INTRODUCCIÓN}

El Ministerio de Educación (MINED), en particular los centros educacionales de la educación media, tiene la responsabilidad de desarrollar un proceso de orientación profesional que brinde las herramientas necesarias para que los estudiantes, una vez llegado el momento, se presenten a un proceso de selección profesional autodeterminado, que responda al contenido psicológico de su personalidad y a las necesidades que demanda el contexto social, aspecto que constituye un reto en la labor educativa del profesor.

El educador es un agente socializador de primera instancia y desde el cumplimiento de sus tareas y funciones debe contribuir al logro de los objetivos generales de la educación, entre ellos, favorecer la educación vocacional y profesional, de forma tal que desarrolle en sus estudiantes conocimientos y vivencias afectivas que sean la base para la formación de intereses cognoscitivos por las diversas profesiones.

Autores como (Álvarez et al., 2018), (García Ancira et al., 2017), (Contreras Pérez et al., 2017) han investigado la orientación profesional al identificar diferentes problemáticas manifiestas en la educación primaria, media básica, media superior y en las universidades, para lo cual se han elaborado diversas propuestas que van desde el accionar cooperado de la escuela con la familia y la fundamentación de los enfoques: problematizador, grupal, artístico, familiar y multifactorial, con la intencionalidad de contribuir al desarrollo de motivaciones, intereses, aspiraciones y proyectos de vidas relacionados con las diferentes profesiones y han demostrado la importancia de los actores sociales y comunitarios en la formación del sujeto como personalidad, en un constante cambio, formación y desarrollo.

Un momento decisivo en el proceso de orientación profesional de los estudiantes lo constituyen los estudios preuniversitarios, donde los adolescentes y jóvenes que asisten a estos centros educativos se preparan, algunos para iniciar estudios universitarios y otros para incorporarse al mundo laboral, razones por las que se deben acercar al mundo de las profesiones, a sus características, particularidades y encargo social, en el trabajo conjunto de la escuela, la familia y la comunidad; de los diferentes agentes y agencias socializadoras. En este sentido, resulta necesario establecer congruencias entre los intereses del estudiante y las demandas profesionales del territorio, cuestiones que en la actualidad se presenta como principal problema.

A pesar de las investigaciones realizadas en pos de lograr un buen proceso de orientación profesional en las escuelas, aun prevalece que la orientación profesional no está dirigida a la formación de expectativas, intereses, aspiraciones y proyectos de vida profesional en los estudiantes por las carreras que son necesarias para el desarrollo local del municipio.

Se plantea lo anterior a partir de los resultados de indagaciones teóricas y empíricas (análisis de documentos, observaciones a la realidad educativa escolar y entrevista) realizadas a los

50 UNESUM-Ciencias. Publicación cuatrimestral. Vol. 6, Año 2022, No. 1 (Enero - Abril) 
estudiantes y profesores de Educación Media, se evidencian insuficiencias como la limitada preparación de los docentes y directivos para la gestión del proceso de orientación profesional, a partir de las necesidades y potencialidades del desarrollo local.

Por tanto la orientación profesional en la enseñanza media es un tema que requiere una solución contextualizada, que este a tono con las carencias e individualidades de los docentes y directivos de nuestro territorio, teniendo en cuenta que son los principales responsables de guiar el proceso de enseñanza- aprendizaje.

Cabe entonces preguntarse: ¿cómo contribuir al desarrollo del proceso de orientación profesional en correspondencia con las necesidades de desarrollo local, en los estudiantes de educación media? Es obvio que se hace necesaria una estrategia pedagógica que contribuya al desarrollo del proceso de orientación profesional en correspondencia con las necesidades de desarrollo local, en los estudiantes de educación media, del municipio Pinar del Río.

\section{DESARROLLO}

\section{Materiales y métodos:}

Por la importancia de las insuficiencias planteadas en la investigación se utilizó los siguientes métodos:

El análisis documental permite la búsqueda de información para conformar el marco teórico conceptual y el análisis histórico de la evolución del objeto de estudio en documentos normativos y metodológicos del preuniversitario como las orientaciones metodológicas, las actas de las asambleas de grupo, los proyectos educativos, entre otros.

La observación, para evaluar cómo se comportan los indicadores de la variable: proceso de orientación profesional en correspondencia con las necesidades de desarrollo local en la constatación del estado inicial del problema, a partir de la observación de actividades docentes y extradocentes, y durante la marcha del pre - experimento, así como el comportamiento de la estrategia pedagógica.

\section{Orientación profesional}

Aunque resulta difícil precisar la fecha de nacimiento de la Orientación, si podemos hacer un recorrido por los hechos y aportaciones más relevantes que dibujan el posible mapa de su concepción y desarrollo y en función del mismo (Ramos Cordero \& Breijo Worosz, 2017).

La Orientación Profesional surge de manera formal a inicios del siglo XX. El término surge en 1909 con los trabajos de Parsons, en sus inicios tendió más hacia los intereses, aptitudes, motivaciones y el conocimiento del contexto (posibilidades de estudio y de trabajo).

Posteriormente, en los años 30, la orientación profesional se enfocó en el desarrollo de habilidades de estudio y en los procesos de aprendizaje (Orientación Educativa); hacia la década del 70 el interés se fijó en una orientación para el desarrollo, enfatizando en la prevención, la adquisición y el desarrollo experto de habilidades para la vida, la educación para la salud, habilidades sociales, la prevención de consumo de drogas, ect. 
La orientación como categoría, existe desde los albores de la humanidad y ha sido definida por investigadores como (Calzadilla Pérez, 2017), (López, 2021), (Fonseca et al., 2019). Su contenido y esencia cambian con el contexto histórico, son determinados objetivamente por las condiciones de vida y el tipo de relaciones sociales imperantes, entre otros factores. El problema de la orientación del hombre hacia las distintas profesiones y oficios no es nuevo, tan pronto como el hombre se desarrolló y los oficios aparecieron, la orientación profesional se convirtió en una tarea de primer orden a cumplir por la sociedad, es un término antiguo, y alcanza hoy una vigencia extraordinaria.

La orientación profesional ha sido objeto de estudio a nivel mundial por diferentes autores, los que, desde perspectivas teóricas, enfoques y rasgos diferentes han incursionado en este proceso tan complejo. Su naturaleza ha variado a través de las diferentes etapas de desarrollo de la humanidad, se haya estrechamente relacionada con los cambios sociales, el papel del trabajo en la sociedad y la forma en que el hombre percibe las distintas profesiones. Al analizar los elementos acerca del origen de la orientación profesional es posible organizar diferentes etapas, que se hallan estrechamente relacionadas con el desarrollo de las ciencias.

(del Carmen Ramírez-González, 2020) plantea cuatro etapas que marcan la evolución de la orientación profesional hasta la actualidad. Estas son:

- Primera etapa: indicios de orientación profesional en el Mundo Antiguo (antes del siglo $\mathrm{XI})$

- Segunda etapa: la orientación hacia los oficios en la Edad Media (siglos XI-XIV)

- Tercera etapa: la orientación profesional en la época moderna (siglos XV-XVIII)

- Cuarta etapa: la orientación profesional en la época contemporánea (a partir del siglo XIX y hasta la actualidad)

El estudio de estas etapas permite constatar la evolución de tan complejo proceso, donde queda demostrado que la actividad de orientación surgió con el mismo hombre, vinculada al proceso de formación y desarrollo de la personalidad, en función de satisfacer diversas necesidades, entre ellas, las relacionadas con la selección, estudio y práctica profesional.

Al respecto (Gómez Ruiz, 2020) plantea que, durante el siglo XX, estas corrientes fueron: Psicométrica, Clínico - Médica, Humanista y la Integrativa. Considera que el surgimiento de estas corrientes está dado por tres aspectos fundamentales: las particularidades y retos del mundo contemporáneo, el desarrollo específico de la educación y la búsqueda de soluciones a los problemas educativos, así como al desarrollo de la Psicología y su aplicación al campo educacional.

Estas corrientes trajeron consigo el desarrollo de diversas investigaciones, que en el campo de la orientación profesional manifestaron la presencia de diferentes enfoques, reflejo de las posiciones de distintos autores acerca del origen de la motivación, intereses y preferencias profesionales del sujeto y cómo esto se expresa en la actividad profesional.

El enfoque de la orientación centrado en la personalidad, en el que se destacaban tres teorías: las teorías psicodinámicas de la orientación profesional (sus principales exponentes fueron Bordin, Nachman y Holland); las teorías no directivas de la orientación profesional (se destacan en la

52 UNESUM-Ciencias. Publicación cuatrimestral. Vol. 6, Año 2022, No. 1 (Enero - Abril) 
segunda mitad del siglo XX y entre sus precursores encontramos a Rogers, C. y Maslow, A. H.) y las teorías evolutivas (sus principales representantes fueron Super, D., Ginzberg, E., O’ Hara).

En criterios de esta autora, las teorías evolutivas se manifestaron con mayor fuerza a partir de los años 50 debido fundamentalmente al desarrollo de la Psicología Humanista.

El análisis de estas posiciones permite plantear que la orientación profesional toma gran auge en la época a partir de estos enfoques, los que devenían en posiciones teóricas, cada uno de ellos constituyen aportes científicos, independientemente de las limitaciones teóricas y metodológicas que pudieran presentar.

De igual forma estos enfoques, corrientes y teorías se extendieron a nivel internacional, a lo que no estaba ajena Cuba, aunque no es hasta la segunda mitad del siglo XX, que en el país se puede hablar de acciones puntuales en el ámbito de la orientación profesional.

A partir del curso escolar 1969 - 1970, se tomaron medidas dirigidas a lograr mayor correspondencia entre las necesidades de la fuerza de trabajo del país y las matrículas universitarias. La orientación profesional estaba dirigida a brindarles información a los estudiantes de las carreras universitarias, con énfasis en las priorizadas, según las necesidades económicas y sociales.

Incorporar otras definiciones, para definir cuál asumes.

Disímiles son las definiciones que constan en la literatura científica de orientación profesional abordadas por diferentes autores como: Laura Domínguez, Pino, Z. Matos, Adis Barbara López Menéndez.

La autora asume en la investigación la definición de (Jiménez, 2017), en relación con el Enfoque Histórico-Cultural que "implica diseñar situaciones de aprendizaje que estimulen la formación y desarrollo de las inclinaciones del sujeto hacia una u otra profesión, así como de su capacidad de autodeterminación profesional. Asumir esta definición, implica que se reconoce que la orientación profesional incluye la formación vocacional de los estudiantes; es un trabajo que debe realizarse de manera coherente, utilizando las vías en correspondencia con las características de los estudiantes de preuniversitario puesto que en este nivel es esencial ofrecer toda la ayuda necesaria para que estos puedan decidir de manera consciente y autoderminada el estudio de la profesión.

Se definido la orientación profesional concebida desde una perspectiva histórico-cultural del desarrollo humano como:

"La relación de ayuda que establece el Orientador Profesional (psicólogo, pedagogo, maestro) con el Orientado (el estudiante) en el contexto de su educación (como parte del proceso educativo que se desarrolla en la escuela, la familia, la comunidad) con el objetivo de propiciar las condiciones de aprendizaje necesarias para el desarrollo de las potencialidades de la personalidad del estudiante que le posibiliten asumir una actuación autodeterminada en el proceso de elección, formación y desempeño profesional." (Santana Sardi \& Vigueras Moreno, 2019).

Concebir la Orientación Profesional como un proceso significa entender que transcurre a lo largo de la vida del sujeto atravesando diferentes momentos o etapas las cuales no se corresponden 
exactamente con determinadas edades o niveles de enseñanza, sino que transcurren en dependencia del nivel de desarrollo de la personalidad logrado por los sujetos y el momento de su inserción en la enseñanza profesional y en la vida laboral, lo que reafirma la necesidad de un trabajo diferenciado de Orientación Profesional.

Los municipios cubanos se conforman como célula de lo local/territorial desde inicios del siglo xx, con la aprobación de la Enmienda Platt al constituirse la República, cuando finaliza la guerra de independencia en el país. La Constitución de 1940 legitima el municipio como base económica territorial y le concede ciertos atributos en el orden administrativo y económico que tratan de garantizar una relativa capacidad financiera propia para dar respuestas a las necesidades en términos de desarrollo, básicamente referidos a la infraestructura.

Los procesos de desarrollo local que comienzan a tomar auge en el país a

partir de finales de la década de 1980 constituyen una forma de solución a las necesidades de los municipios los cuales se articulan y complementan con la estrategia de desarrollo nacional del país.1 La sistematización y generalización de estas experiencias por parte de los académicos cubanos va conformando una visión cubana propia del desarrollo local, que se nutre de la teoría del desarrollo endógeno local -proveniente principalmente de Europa- e incorpora las especificidades de estos procesos en un país con las condiciones y retos específicos de Cuba.

El proceso de actualización del modelo económico cubano, lo que ha impulsado las iniciativas de desarrollo municipal; con lo cual no solo se pretende revitalizar la dimensión local/municipal de los procesos de desarrollo,2 sino también perfeccionar el modelo de gestión territorial cubano, otorgarles a los municipios y a sus gobiernos la necesaria descentralización en los marcos de una economía centralmente planificada, y desarrollar los instrumentos que posibiliten la aplicación integral de la concepción del desarrollo local y que permitan dar solución a las contradicciones que se sintetizan en el municipio como célula económica, o que se derivan del propio proceso en curso.

La recepción de la teoría sobre el desarrollo local -proveniente principalmente de fuentes europeas, en las que se referencian básicamente los trabajos de autores como (Ormaza-Mejía, 2019), entre otros -se produce en medio de una fuerte polémica de carácter ideológico acerca de la viabilidad del desarrollo local para un país con las particularidades de Cuba. No es hasta la entrada del país en el Periodo Especial, en la década de 1990, que se debate en los medios académicos cubanos la viabilidad de esta forma de desarrollo para un país subdesarrollado, con un proyecto de construcción socialista y un alto nivel de centralización en la toma de decisiones y la distribución de los recursos.

Considerando las particularidades propias de Cuba como país subdesarrollado, una definición de desarrollo local debe contener los siguientes aspectos:

1. Las iniciativas de desarrollo local son promovidas de forma conjunta por todos los actores políticos y sociales bajo la dirección del Partido y el Gobierno.

2. Se promueven actividades económicas eficientes y eficaces que incidan decisivamente en el sector productivo y en el incremento de los valores productivos.

3. Las actividades están encaminadas al incremento de la efectividad social y del bienestar de la población.

54 UNESUM-Ciencias. Publicación cuatrimestral. Vol. 6, Año 2022, No. 1 (Enero - Abril) 
En síntesis, los procesos de desarrollo local en el país deben estar articulados a la estrategia de desarrollo trazada por él; dar respuesta a los problemas originados a partir de la persistencia de desigualdades territoriales y de la necesidad de garantizar la soberanía alimentaria del país; y transformar su estructura productiva en función de elevar el nivel y calidad de vida del ser humano, elemento rector y central de dicha estrategia de desarrollo.

A consideración de Carlos César Torres el desarrollo local es un "proceso de construcción social y cambio estructural que desde un entorno innovador territorial desarrolla capacidades locales para gestionar políticas públicas, estrategias, programas y proyectos orientados a aprovechar recursos endógenos y exógenos y articular armónicamente intereses nacionales, sectoriales y territoriales, fomentando transformaciones económicas, sociales, naturales y político institucionales en las localidades sobre bases sostenibles y con una activa y protagónica participación ciudadana, en función de elevar la calidad de vida de la población”.(Martínez Clares \& González Lorente, 2018)

$\mathrm{Al}$ referirse entonces a la perspectiva histórico cultural que asumen la orientación profesional y el desarrollo local, centrados esencialmente en la comprensión de los fenómenos que lo rodean y que contribuyen a su existencia, a las situaciones socioculturales que emanan de su relación y a la reflexión crítica que aparece en su conceptualización, se manifiesta un estrecho vínculo, donde la persona que orienta aparece como protagonista y gestor de este proceso de orientación profesional.

\section{CONCLUSIONES}

La investigación radica en proponer un proceso de orientación profesional en correspondencia con las necesidades de desarrollo local, combinado con la necesidad de contribuir a la formación de intereses, aspiraciones, motivaciones y proyectos en los estudiantes, por las profesiones que son imprescindibles para el desarrollo del municipio, en las dimensiones económico-productiva, político-social y educativo-cultural. Posibilita la implementación de una estrategia pedagógica en la práctica del proceso de orientación profesional en correspondencia con las necesidades de desarrollo local, en los estudiantes de la Educación Media y que comprende un sistema de acciones por etapas.

\section{REFERENCIAS BIBLIOGRÁFICAS}

Álvarez, P. R., Gómez, C. I. O., \& Campos, J. A. S. (2018). Valoración de la orientación profesional en la enseñanza secundaria postobligatoria. Revista de Investigación Educativa, 36(1), 75-91. https://revistas.um.es/rie/article/download/285881/221531

Calzadilla Pérez, O. O. (2017). La integración de la Orientación Profesional Pedagógica en la asignatura Didáctica de la Lengua Española de la carrera Licenciatura en Educación Primaria. Voces y silencios. Revista $\begin{array}{llll}\text { Latinoamericana de } & \text { Educación, } & \text { 82-102. }\end{array}$ https://revistas.uniandes.edu.co/doi/pdf/10.18175/vys8.1.2017.06

Contreras Pérez, J. M., Mirabal Díaz, J. M., Fong Zurbano, G. L., Machado Contreras, M. D., de la Hoz Rojas, L., \& Cobo Lueje, M. d. R. (2017). Visita virtual a la Facultad de Estomatología: pertinencia en la orientación profesional de la carrera. EDUMECENTRO, 9(3), 232-248. http://scielo.sld.cu/scielo.php?pid=S2077$\underline{28742017000300015 \& \text { script }=\text { sci_arttext\&tlng }=\text { en }}$ 
del Carmen Ramírez-González, M. (2020). Orientación profesional para el tratamiento a escolares con $\begin{array}{lllll}\text { manifestaciones } \quad \text { de } & \text { agresividad. } & \text { 113-121. }\end{array}$ https://www.redalyc.org/journal/5891/589164533011/html/

Fonseca, D. B. R., Morales, Y. G., \& Llópis, P. E. A. (2019). LA ORIENTACIÓN PROFESIONAL. UN RETO EDUCATIVO. Didasc@ lia: didáctica y educación ISSN 2224-2643, 10(6), 91-103. https://revistas.ult.edu.cu/index.php/didascalia/article/download/1085/1074

García Ancira, C., Castillo Elizondo, J. A., \& Salinas Reyna, I. M. (2017). El seguimiento a egresados como orientación profesional para estudiantes y aspirantes a las carreras de ingeniería. Revista Cubana de Educación Superior, 36(3), 63-73. http://scielo.sld.cu/scielo.php?script=sci_arttext\&pid=S025743142017000300006

Gómez Ruiz, A. E. (2020). Análisis del proceso de orientación profesional en el bachillerato, de los estudiantes del primer semestre de la carrera de Economía de la Universidad Técnica del Norte http://repositorio.utn.edu.ec/bitstream/123456789/10411/5/05\%20FECYT\%203648\%20TRABAJO\%20GR $\underline{\text { ADO.pdf }}$

Jiménez, D. P. (2017). LA ORIENTACIÓN PROFESIONAL PEDAGÓGICA DESDE EL PROCESO DE EXTENSIÓN UNIVERSITARIA EN EL CUM TAGUASCO. Márgenes, 5(1), 16-30. https://revistas.uniss.edu.cu/index.php/margenes/article/download/365/520

López, M. J. D. (2021). La perspectiva de género en instituciones de formación. Propuestas para la orientación profesional. Cuaderno de Pedagogía Universitaria, 18(36), 115-127. https://dialnet.unirioja.es/descarga/articulo/8078200.pdf

Martínez Clares, P., \& González Lorente, C. (2018). Orientación, empleabilidad e inserción laboral en la universidad a través de un Modelo de Ecuaciones Estructurales/Career guidance, employability and entering the workforce at University through a Structural Equation Model. Revista española de pedagogía, 119-139. https://dialnet.unirioja.es/descarga/articulo/6276780.pdf

Ormaza-Mejía, P. (2019). Educación: Orientación Vocacional y Profesional, garantía de derechos y construcción de $\begin{array}{lllll}\text { proyectos de } & \text { Revista Ciencia UNEMI, } & 12(30),\end{array}$ https:/www.redalyc.org/jatsRepo/5826/582661249010/582661249010.pdf

Ramos Cordero, Y., \& Breijo Worosz, T. (2017). Acercamiento histórico a la orientación profesional hacia las especialidades técnicas desde la secundaria básica. Mendive. Revista de Educación, 15(2), 242-252. http://scielo.sld.cu/scielo.php?script=sci_arttext\&pid=S1815-76962017000200011

Santana Sardi, G. A., \& Vigueras Moreno, J. A. (2019). Hacia un Sistema Virtual de orientación vocacional. Revista Cubana de Educación Superior, 38(3). http://scielo.sld.cu/scielo.php?script=sci_arttext\&pid=S0257$\underline{43142019000300011}$ 